\title{
Pregabalin effects on neural response to emotional faces
}

\section{Robin L. Aupperle 1,3,46*, Dharol Tankersley ${ }^{1,78}$, Lakshmi N. Ravindran ${ }^{5}$, Taru Flagan ${ }^{1}$, Nathan R. Stein ${ }^{1}$, Murray B. Stein ${ }^{1,2,4}$ and Martin P. Paulus ${ }^{1,4}$}

\author{
' Department of Psychiatry, University of California, San Diego, La Jolla, CA, USA \\ 2 Department of Family and Preventive Medicine, University of California, San Diego, La Jolla, CA, USA \\ ${ }^{3}$ Mental IIIness Research, Education, and Clinical Center, Veterans Affairs San Diego Healthcare System, La Jolla, CA, USA \\ ${ }^{4}$ Psychiatry Service, Veterans Affairs San Diego Health Care System, La Jolla, CA, USA \\ ${ }^{5}$ Department of Psychiatry, University of Toronto, Toronto, ON, Canada \\ ${ }^{6}$ Department of Psychology, University of Missouri, Kansas City, MO, USA \\ 7 Computational Psychiatry Unit, Baylor College of Medicine, Houston, TX, USA \\ ${ }^{8}$ Schipul Technologies, Houston, TX, USA
}

\section{Edited by:}

Hans-Jochen Heinze, University of

Magdeburg, Germany

\section{Reviewed by:}

Ruthger Righart, Institute for Stroke and Dementia Research, Germany Catherine Harmer, University Department of Psychiatry, UK David Nutt, Imperial College London, UK

\section{*Correspondence:}

Robin L. Aupperle, Department of Psychology, University of Missouri Kansas City, 5030 Cherry Street, Cherry Hall Room 301, Kansas City, MO 64110, USA.

e-mail: aupperler@umkc.edu

Pregabalin has shown promise in the treatment of anxiety disorders. Previous functional magnetic resonance imaging (fMRI) studies indicate agents used to treat anxiety, e.g., SSRIs and benzodiazepines, attenuate amygdala, insula, and medial prefrontal cortex (mPFC) activation during emotional processing. Our prior study has shown that during anticipation of an emotional stimulus, pregabalin attenuates amygdala and insula activation but increases medial PFC activation. In this study, we examined whether, similar to SSRIs and benzodiazepines, pregabalin attenuates amygdala, insula, and medial PFC during emotional face processing. Sixteen healthy volunteers underwent a double-blind within-subjects fMRI study investigating effects of placebo, $50 \mathrm{mg}$, and $200 \mathrm{mg}$ pregabalin on neural activation during an emotional face-matching task. Linear mixed model analysis revealed that pregabalin dose-dependently attenuated left amygdala activation during fearful face-matching and left anterior insula activation during angry face-matching. The $50 \mathrm{mg}$ dose exhibited more robust effects than the $200 \mathrm{mg}$ dose in the right anterior insula and ventral ACC. Thus, pregabalin shares some similarity to SSRIs and benzodiazepines in attenuating anger and fear-related insula and amygdala activation during emotional face processing. However, there is evidence that a subclinical $50 \mathrm{mg}$ dose of pregabalin produced more robust and widespread effects on neural responses in this paradigm than the more clinically relevant $200 \mathrm{mg}$ dose. Taken together, pregabalin has a slightly different effect on brain activation as it relates to anticipation and emotional face processing, which may account for its unique characteristic as an agent for the treatment of anxiety disorders.

Keywords: anxiety, anxiolytic, benzodiazepine, emotion, fMRI, pharmaco-imaging, pregabalin

\section{INTRODUCTION}

Anxiety disorders affect approximately one in five adults each year in the United States (Kessler et al., 2005) and are associated with significant psychological and somatic distress as well as increased healthcare costs for those afflicted (Hoffman et al., 2008; Bereza et al., 2009; Johnston et al., 2009; Pirkola et al., 2009). First line pharmacologic therapies include selective serotonin reuptake inhibitors (SSRIs) and benzodiazepines. However, SSRIs are associated with side effects such as weight gain and sexual dysfunction, usually take 2-4 weeks to effect symptom reduction, and often fail to produce full remission (Baldwin, 2008). Benzodiazepines have a more immediate effect on symptoms, but are associated with concerns regarding side effects such as sedation and cognitive impairment, as well as potential risks for abuse or dependence (McIntosh et al., 2004; Cloos and Ferreira, 2009). Considering these issues, there remains considerable room to improve both the effectiveness and side effect profile of pharmacologic anxiety treatment.

Pregabalin, currently marketed in the US for treatment of fibromyalgia and neuropathic pain, has also shown promise as an anxiolytic. Pregabalin has demonstrated anxiolytic effects in acute paradigms (i.e., those investigating short-term effects), such as presurgical anxiety (Nutt et al., 2008), and in the chronic treatment of generalized anxiety and social anxiety disorder (Pande et al., 2004; Rickels et al., 2005; Tassone et al., 2007). This drug appears to have a faster onset of anti-anxiety effects than SSRIs and fewer side effects and lower rates of discontinuation due to adverse events than benzodiazepines (Feltner et al., 2003; Pande et al., 2003, 2004; Hindmarch et al., 2005; Pohl et al., 2005; Rickels et al., 2005; Montgomery et al., 2006; Oulis et al., 2008; Lydiard et al., 2010). Benzodiazepines are thought to exert anxiolytic effects through modulation of GABAergic neurotransmission (Atack, 2003) and SSRIs are thought to exert effects primarily through modulation of the 5HT system (Vaswani et al., 2003). PGB's primary mechanism of action is thought to involve selective binding at the $\alpha_{2} \delta$ subunit of voltage-dependent calcium channels, subsequently leading to upregulation of GABA inhibitory activity and a reduction in the release of several neurotransmitters, including glutamate, noradrenaline, serotonin, and substance P (Fink et al., 2002; Dooley et al., 2007; Brawek et al., 2008). The anxiolytic effects observed for PGB may, therefore, relate to its eventual effects on neurotransmitter systems (e.g., serotonin, GABA) that have been implicated in 
anxiety disorders and are influenced by other classes of anxiolytic medications (e.g., benzodiazepines, SSRIs) (Lydiard, 2003; Millan, 2003; Gordon and Hen, 2004; Lowry et al., 2005; Ebner and Singewald, 2006; Ravindran and Stein, 2010; Kalk et al., 2011). The molecular distinction between pregabalin and the other agents (i.e., selective action at voltage-dependent calcium channels) might explain the selective clinical profile observed with pregabalin (i.e., fewer side effects).

Functional magnetic resonance imaging (fMRI) and other neuroimaging methodologies have been useful for understanding the neurobiology of anxiety disorders. During symptom provocation or processing of emotional stimuli, individuals with anxiety disorders have been reported to exhibit enhanced responsivity within a neural circuitry centering on the medial prefrontal cortex (mPFC), anterior cingulate cortex (ACC), insula, and amygdala (Cannistraro and Rauch, 2003; Etkin and Wager, 2007; Shin and Liberzon, 2010), though there is some variability and/or additional findings depending on the disorder and the particular paradigm studied. The amygdala has been implicated in the processing of salience, particularly in response to fear-related stimuli (Davis and Whalen, 2001; Hariri and Whalen, 2011; Kim et al., 2011), while the insula is thought to be important for monitoring and predicting changes in interoceptive state (i.e., internal bodily state) in response to affective stimuli (Paulus and Stein, 2006; Knutson and Greer, 2008; Craig, 2011). Medial PFC and ACC regions are considered important in the integration of affective information, regulating brain and behavioral responses, and monitoring conflict and errors (Ochsner and Gross, 2005; Botvinick, 2007; Kanske et al., 2010; Etkin et al., 2011). In anxiety disorders, prefrontal regions are theorized to play a role in the regulation of limbic (e.g., amygdala) activation and in determining the cognitive and behavioral responses to emotional stimuli (Aupperle and Paulus, 2010; Hofmann et al., 2011; Kim et al., 2011).

It has been proposed that fMRI may also be a viable method for characterizing neural mechanisms of pharmacologic treatments and as a biomarker for predicting anxiolytic function for novel agents (Paulus and Stein, 2007). Thus, studies have begun to investigate the effects of pharmacologic agents on activation within the prefrontal-insula-amygdala neural circuitry implicated in anxiety. Much of this research has focused on neural effects of anxiolytic agents in healthy non-anxious populations, as this method would be most efficient if used as a biomarker for testing viability of novel pharmacologic agents. fMRI studies with healthy controls report acute benzodiazepine administration to be associated with attenuated activation of insula, amygdala, and $\mathrm{mPFC}$ during paradigms involving processing of emotional faces (Paulus et al., 2005), anticipation of painful stimuli (Wise et al., 2007), and punishment during risk-based decision-making (Arce et al., 2006). Sub-chronic SSRI administration (e.g., 3-30 days) has been associated with decreased amygdala, insula, and/or $\mathrm{mPFC}$ and ACC activation during anticipation (Simmons et al., 2009) and processing of aversive pictures (Takahashi et al., 2005), and masked and unmasked emotional faces (Del-Ben et al., 2005; Harmer et al., 2006; Anderson et al., 2007; Arce et al., 2008; Windischberger et al., 2010).

We previously reported pregabalin to dose-dependently decrease activation within insula and amygdala and increase medial
PFC activation during anticipation of emotional images (Aupperle et al., 2011). This study provided initial support for the notion that anxiolytics, regardless of the molecular mechanism of action or the direction of influence on prefrontal regions, have the effect of attenuating amygdala and insula responses during emotional processing. However, the consistency of pregabalin's effect on limbic-prefrontal response patterns for different fMRI paradigms has not yet been investigated. In the current study, we sought to investigate whether our previous findings regarding pregabalin's effects on neural responses during emotional anticipation would generalize to emotional face processing, which has been extensively investigated in relation to limbic function, fear, and anxiety disorders (Adolphs, 2008; Fusar-Poli et al., 2009; Staugaard, 2010). We hypothesized that pregabalin would be associated with decreased responses within the amygdala and insula specifically during negative (i.e., angry and fearful) emotional face processing.

\section{MATERIALS AND METHODS PARTICIPANTS AND STUDY DESIGN}

Sixteen right handed, healthy volunteers (10 male; mean age $=$ $23.2 \pm 2.6$ years; nine Caucasian) participated in three scanning sessions in which they were administered placebo, $50 \mathrm{mg}$, or $200 \mathrm{mg}$ doses of pregabalin. This same cohort of subjects has been reported on previously with a different paradigm (Aupperle et al., 2011). The specified doses of PGB were chosen in order to investigate a dose shown to be clinically relevant $(200 \mathrm{mg})$ as compared to a lower dose shown to not produce noticeable side effects but to not be as clinically effective $(50 \mathrm{mg}$ ) (Feltner et al., 2003). There was a 1-2 week delay between each test session to allow for sufficient elimination of the study drug. The assignment of placebo or study drug for each session was determined in a pseudo-randomized order and was administered in a double-blind fashion. Diagnostic interviews were conducted and exclusion criteria included satisfying current or life-time DSM-IV criteria for Axis I or II disorders, current substance abuse or medications, neurological disorders, smoking, or excessive caffeine consumption ( $>4$ cups/day). All subjects gave written, informed consent, and were treated in accordance with the Declaration of Helsinki. The study was approved by the Human Research Protection Program of the University of California, San Diego.

Subjects performed an event-related version of an emotional face-matching task adapted from that described by Hariri et al. (2002) and using emotional faces from the standard NimStim Set of Facial Expressions (Tottenham et al., 2009) and from Matsumoto and Ekman (Matsumoto and Ekman, 1988). An event-related design was used to enable investigation of neural responses when matching each different face type (happy, fearful, and angry). During each $5 \mathrm{~s}$ trial, a target face (on the top of the computer screen) and two probe faces (on the bottom of the screen) were presented, and subjects were instructed to match the probe with the same emotional expression as the target by pressing the corresponding left or right button. During a control condition, subjects were instructed to match the target shape to one of two differently oriented probe shapes. The interstimulus interval was the period between the subject's response and the end of the $5 \mathrm{~s}$ trial. The face and shape trials used in this paradigm are illustrated in Figure 1. 


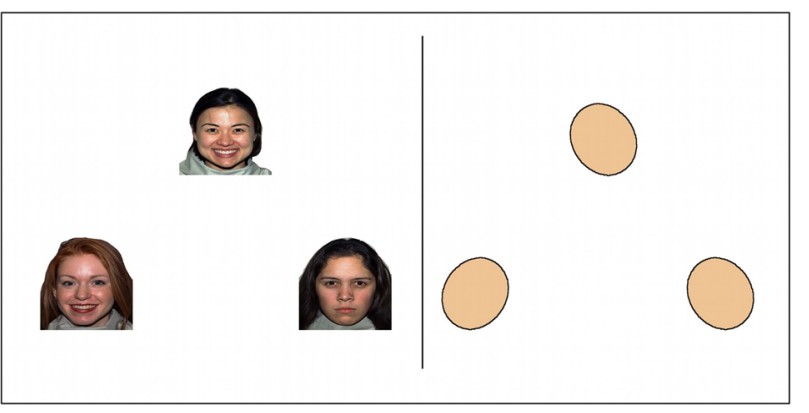

FIGURE 1 | fMRI emotional face matching task. This task was adapted from the block-design task described by Hariri et al. (2002) to an event-related design using the emotional face images from Tottenham et al (2009) and Matsumoto and Ekman (1988). This task includes conditions requiring the subject to match emotional faces or shapes. For the faces conditions, a target face (on the top of the computer screen) and two probe faces (on the bottom of the screen) are presented, and subjects are to match the probe with the same emotional expression to the target by pressing the corresponding button. For the faces conditions, the subjects are matching an equal number of angry, fearful, and happy emotional faces. During the control shape condition, subjects are to match the target shape to one of two differently oriented probe shapes.

Subjects performed two 8-min runs, each consisting of 24 each of shapes, happy, sad, and fearful face-matching conditions. Trial types were presented in a pseudorandom order. Prior to dosing, subjects completed the Karolinska Sleepiness Scale (KSS) (Akerstedt and Gillberg, 1990), State-Trait Anxiety InventoryState version (STAI-T) (Spielberger et al., 1983), and rated their current state on a Visual Analog Scale (VAS) on the following dimensions: anxious, embarrassed, tense, tired, dizzy, "high," nervous and trembling. After completing these baseline measures, oral doses of pregabalin or placebo were administered with a small amount of diet coke. Scanning began approximately $1 \mathrm{~h}$ after dosing. Plasma drug concentrations were acquired every $30 \mathrm{~min}$ throughout the session. Subjects completed KSS, STAI-S, and the VAS measures again after the scan.

\section{DATA ACOUISITION}

Images were acquired on a 3T GE CXK4 scanner. High-resolution T1-weighted sagitally acquired anatomical images (SPGR, TR = $20 \mathrm{~ms}, \mathrm{TE}=4.8 \mathrm{~ms}, \mathrm{FOV}=25 \mathrm{~cm}$, approximately $1.5 \mathrm{~mm}^{3}$ voxels, 124 slices) were obtained. During the task, whole-brain BOLD images were acquired with an 8 -channel array coil (T2* weighted, axially-acquired EPI, $3.43 \times 3.43 \times 2.6 \mathrm{~mm}^{3}$ voxels with a $1.4 \mathrm{~mm}$ gap, $\mathrm{TR}=2000 \mathrm{~ms}, \mathrm{TE}=32 \mathrm{~ms}$, flip angle $=$ 90, 30 slices). Whole-brain images sensitive to arterial spin labeling (ASL) were acquired with a PICORE-QUIPSS II (Wong et al., 1998) sequence with dual-echo spiral acquisition (TE1 = $3 \mathrm{~ms}, \mathrm{TE} 2=30 \mathrm{~ms}), \mathrm{TR}=2000 \mathrm{~ms}$, flip angle $=90$ degrees, $3.43 \times 3.43 \mathrm{~mm}$ in-plane resolution, five $6 \mathrm{~mm}$ slices, $20 \mathrm{~cm}$ tag width, $1 \mathrm{~cm}$ gap between the tag and the proximal imaging slice, TI1 $=700 \mathrm{~ms}$, TI2 $=1400 \mathrm{~ms}$. ASL scans were calibrated between subjects and scanning sessions as described in (Floyd et al., 2003). Proton density of cerebrospinal fluid, a signal index directly proportional to blood and relatively consistent across time and subjects, was obtained with a single image through the ventricles at $\mathrm{TE}=3 \mathrm{~ms}$. ASL scans were included in the current study to identify pregabalin's overall effects on cerebral blood flow $(\mathrm{CBF})$ and to determine whether pregabalin effects on $\mathrm{fMRI} B O L D$ response related to the overall CBF effects.

\section{BEHAVIORAL AND PLASMA DATA ANALYSIS}

Linear mixed effects (LME) models were used to examine the effect of pregabalin dose on behavior, self-report ratings, and plasma concentrations. LMEs were implemented in $\mathrm{R}$ (www.cran.org), which estimates the parameters of the mixed model using the Maximum Likelihood Estimation Procedure. Behavioral data obtained during the scan (CPT reaction time and accuracy) were subjected to LME analyses with subject as random effects and pregabalin dose (placebo, $50 \mathrm{mg}, 200 \mathrm{mg}$ pregabalin) and task valence (matching of fearful vs. happy and angry vs. happy faces) as fixed effects to examine main and interaction effects of each fixed factor. Survey data (KSS, STAI, and VAS scales) were subjected to LME analyses with subject as random effects and pregabalin dose and time of self-report (prior to dosing vs. post-scan) as fixed effects in order to examine the dose by time interaction. To enable further characterization of the effects observed for dose main effects and dose by valence interaction effects, post-hoc linear mixed models were conducted with specific contrasts (e.g., PLB vs. $50 \mathrm{mg}$, PLB vs. $200 \mathrm{mg}, 50 \mathrm{mg}$ vs. $200 \mathrm{mg}$ ), which were considered significant at the level of $p<0.05$.

Plasma concentrations of pregabalin were assessed at National Medical Services Lab using high performance liquid chromatography [HPLC]. The detection limit of this procedure is $0.10 \mathrm{mcg} / \mathrm{mL}$. LME analysis was conducted with subject as random effects and PGB dose and time of blood draw as fixed effects in order to examine the main and interaction effects of dose and time on plasma concentration levels.

\section{fMRI BOLD ANALYSIS}

Data were preprocessed and analyzed using Analysis of Functional NeuroImages (AFNI) software package (Cox, 1996) and $R$ statistical package ( $r$-project.org). All EPI images were aligned to the high-resolution anatomical images and resampled to a voxel size of $64 \mu \mathrm{L}$ or $4 \times 4 \times 4 \mathrm{~mm}$ (from the original $3.7 \times 3.7 \times 4 \mathrm{~mm}$ ). Data were temporally smoothed, spatially blurred with a $4 \mathrm{~mm}$ FWHM spatial filter, and normalized to Talairach space (via AFNI's auto Talairach program, followed by visual inspection of each structural image). A signal to noise ratio (SNR) was calculated as the mean signal within amygdala regions of interest (ROIs) divided by the standard deviation of the mean voxel-wise signal over time. An SNR greater than two standard deviations below the sample SNR was used as a cutoff for excluding runs with poor data quality. One of the two runs for six different scans was, therefore, excluded from the analysis (leaving one run from those scans to be included in the analysis). In total, one run each was excluded for three $50 \mathrm{mg}$ sessions, one placebo session, and two $200 \mathrm{mg}$ sessions.

Preprocessed time-series data for each individual were analyzed using a multiple regression model, based on a BOLD hemodynamic response function with a $4-6$ s peak. Regressors of interest included three orthogonal regressors used to quantify the neural activation related to each task component: (1) matching of 
happy faces, (2) matching of fearful faces, (3) matching of angry faces. Five regressors of no interest were entered into the linear regression model: three motion-related regressors (for roll, pitch, and yaw directions), data from a white-matter mask (averaged at each TR) to control for physiological noise, and a regressor for linear trends used to eliminate slow signal drifts.

Percent signal change (PSC) was calculated by dividing the regressor of interest by the control regressor. The trials involving matching of shapes were not modeled specifically as a regressor of interest but were included as baseline. Therefore, PSC for all conditions of interest (happy, fearful, angry face-matching) were compared to an implicit baseline that included both shapematching and fixation trials. PSC for happy, angry, and fearful conditions were subjected to LME analysis in $R$ (www.cran.org). Separate analyses were conducted to examine task effects and PGB dose effects on neural activation. First, task effects were identified within the placebo-treated group only using LMEs with condition (happy vs. fearful; happy vs. angry faces) as fixed effects and subject as random effects. Second, PGB dose by valence effects were examined using an LME with condition (happy vs. fearful; happy vs. angry faces), and dose (placebo, $50 \mathrm{mg}, 200 \mathrm{mg}$ ) as fixed effects and subject as random effects. Third, main effects of dose on neural activation during each condition type (matching of happy, fearful, and angry faces) were examined by including dose (placebo, $50 \mathrm{mg}, 200 \mathrm{mg}$ pregabalin) as fixed effects and subject as random effects. Finally, an analysis was conducted with test session number as a covariate to determine whether drug-induced changes were partially due to the number of sessions an individual had been in the scanner.

Analyses were conducted voxel-wise across the whole-brain as well as for specific a priori ROIs using small volume correction. Bilateral amygdala, mPFC, and anterior insula were selected as a priori ROIs based on previous literature implicating them in emotional processing, anticipation, and anxiety disorders. The fusiform gyrus was included as an a priori region based on previous literature implicating it to play a primary role in face processing. These anatomical regions were defined in AFNI using the ROI plugin and Talairach Atlas (Lancaster et al., 2000) and are displayed in Figure 2 . The $\mathrm{mPFC}$ included primarily the ventral and dorsal ACC; including BA 32, 24, spreading into ventromedial PFC regions (BA 10). This $\mathrm{mPFC}$ region was partitioned into dorsal and ventral extents at $z=0$, and the anterior insula was partitioned from the insula as a whole using a linear interpolation for the location of the middle insular gyrus. Thus, in total there were eight a priori ROIs (bilateral amygdala, dorsal and ventral medial PFC, bilateral anterior insula, and bilateral fusiform gyrus). A threshold adjustment method based on Monte-Carlo simulations (conducted via AFNI's program Alpha Sim) was used to guard against identifying false positive areas of activation. This resulted in the following minimum volumes for a cluster to be considered significant: $192 \mu \mathrm{L}$ (3 voxels) for the amygdala ROIs, $256 \mu \mathrm{L}$ ( 4 voxels) for anterior insula ROIs, $320 \mu \mathrm{L}$ (5 voxels) for medial PFC ROIs, and $832 \mu \mathrm{L}$ (13 voxels) for whole-brain analyses. While the cluster significance of $p<0.05$ (two-tailed) was used for all analyses, the corrected voxel-wise probabilities (determined via AFNI's program Alpha Sim) are as follows: amygdala $p<0.0021$, anterior insular $p<$

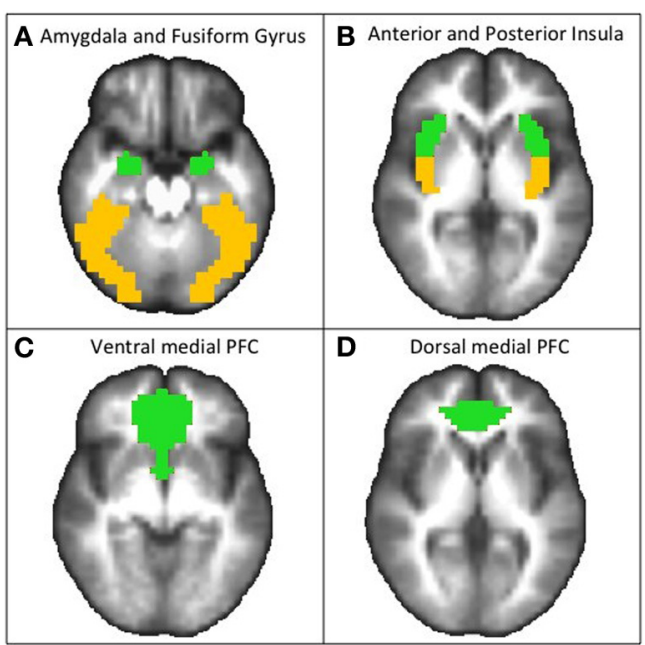

FIGURE 2 | Regions of interest masks used for fMRI analyses, shown from axial views. (A) bilateral amygdala (green) and fusiform gyrus (yellow) (shown at $z=-20$ ), (B) bilateral anterior (green) and posterior (yellow) insula (shown at $z=2$ ), (C) ventral medial prefrontal cortex (PFC; shown at $z=-6$ ), and (D) dorsal medial PFC (shown at $z=4$ ). These anatomical regions were defined using Talairach Atlas [Lancaster et al. (2000)].

0.0015 , posterior insula $p<0.0016$, ventromedial PFC and dorsomedial PFC $p<0.0008$, whole-brain $p<0.00001$. All reported coordinates are center of mass Talairach coordinates $(x, y, z)$ and labeled based on visual observation and Talairach Daemon software (Lancaster et al., 2000). For identified activation clusters, mean $F$-values were extracted and reported. To enable further characterization of the effects, PSC was extracted for each activation cluster and post-hoc linear mixed models were conducted with specific contrasts (e.g., PLB vs. $50 \mathrm{mg}$, PLB vs. $200 \mathrm{mg}$, $50 \mathrm{mg}$ vs. $200 \mathrm{mg}$ ), which were considered significant at $p<0.05$. Spearman's non-parametric correlations were used to investigate potential relationships between extracted PSC for significant clusters and variables that may have influenced BOLD response including (1) fMRI behavioral data and self-report survey data significantly influenced by pregabalin (e.g., fMRI paradigm reaction time, KSS, and VAS ratings of dizziness, tired, trembling, and high) and (2) CBF data collected through ASL (extracted for each ROI activation cluster identified as significant in fMRI analysis). These correlations were considered significant at $p<0.05$.

\section{fMRI ASL ANALYSIS}

ASL scans were reconstructed and CBF was calculated using inhouse scripts in MATLAB (Rack-Gomer et al., 2009). Voxel-based linear mixed models were completed in $R$ to examine potential drug effects on CBF. Whole-brain and anatomically constrained ROI analyses were conducted with these results as described in the previous section for BOLD data.

\section{RESULTS \\ PLASMA CONCENTRATION OF PREGABALIN}

Plasma concentrations of this sample have been reported on previously (Aupperle et al., 2011). Briefly, LME analyses revealed significant effects of dose $[F(1,116)=250.33, p<0.001]$, time 
$[F(4,116)=14.55, p<.001]$, and dose by time interaction $[F(4,116)=11.69, p<0.001]$ on pregabalin plasma concentrations. This indicates that greater dose was associated with greater plasma concentration levels, that concentration levels increased with time, and that there was a greater increase in plasma concentration levels for the $200 \mathrm{mg}$ than the $50 \mathrm{mg}$ dose.

\section{ANALYSIS OF BEHAVIORAL AND SELF-REPORT MEASURES}

Pregabalin effects on self-report measures for this cohort have been reported previously (Aupperle et al., 2011). Briefly, LME modeling revealed a significant dose by time (pre-dosing vs. postscan) effect on ratings of sleepiness $[\mathrm{KSS} ; F(2,72)=3.30, p=$ 0.043; post-hoc analyses revealed $(\mathrm{PLB}=50)<200]$ and visual analogue scale (VAS) ratings of feeling dizzy $[F(2,73)=10.60$, $p<0.001$; post-hoc analyses revealed $(\mathrm{PLB}=50)<200]$, trembling $[F(2,73)=3.74, p=0.028$; post-hoc analyses revealed $50<$ $200]$, high $[F(2,72)=3.19, p=0.047$; post-hoc analyses revealed PLB $<200]$, and tired $[F(2,73)=3.13, p=0.050$; post-hoc analyses revealed $50<200]$. Dose had no significant effect on predosing vs. post-scan ratings of state anxiety [State-Trait Anxiety Inventory-State subscale (STAI); $F(2,73)=2.52, p=0.088$ ], or VAS ratings related to feeling embarrassed $[F(2,73)=1.54, p=$ $0.220]$, nervous $[F(2,73)=1.08, p=0.350]$, anxious, or tense $($ Fs $<1)$.

Subjects responded with high accuracy [mean $=99 \% \pm$ 0.05 standard deviation (SD) of the mean] and quickly (mean response time $=1.33 \mathrm{~s} \pm 0.31 \mathrm{SD}$ ) during all sessions and all four trial types of the emotional face and shape matching task. LME analyses investigating effects of condition (Face vs. Shape; Angry vs. Happy; Fear vs. Happy) and dose on accuracy revealed that pregabalin was associated with slightly higher accuracy $[F(1,171)=4.66 ; p=0.011 ; 200>$ PLB $]$, though this seemed driven by one subject exhibiting slightly lower accuracy during their placebo scan (while all others were 100\% accurate). There was no effect of condition on accuracy; Shape vs. Face: $[F(2,171)=0.049, p=0.825]$; Happy vs. Angry: $F(1,75)=$ $0.016, p=0.901$; Happy vs. Fear: $F(1,75)=0.18, p=0.672$ nor was there a significant dose by condition interaction effect on accuracy; Face vs. Shape: $F(2,171)=0.02, p=0.981$; Happy vs. Angry: $F(2,75)=0.08, p=0.924$; Happy vs. Fear: $[F(2,75)=$ $0.18, p=0.835]$. Pregabalin was associated with slower reaction time across all conditions $[F(1,171)=5.54, p=0.005 ;(200=$ $50)>$ PLB]. There was also a significant difference in reaction time across conditions, with responses being slower to faces than shapes $[F(1,171)=211.62, p<0.001]$, slower to angry than happy faces $[F(1,75)=52.72, p<0.001]$, and slower to fearful than happy faces $[F(1,75)=39.68, p<0.001]$. There was no dose by condition interaction effects on reaction time [Face vs. Shape: $F(2,171)=0.80 ; p=0.451$; Happy vs. Angry: $F(2,75)=$ $0.004, p=0.996$; Happy vs. Fear: $F(2,75)=0.07, p=0.930]$. See Figure 3 for graphical representation of reaction time across conditions and doses.

\section{fMRI ANALYSIS}

\section{Task effects on BOLD response}

Within ROIs, fearful face-matching was associated with greater activation than happy face-matching within the bilateral fusiform

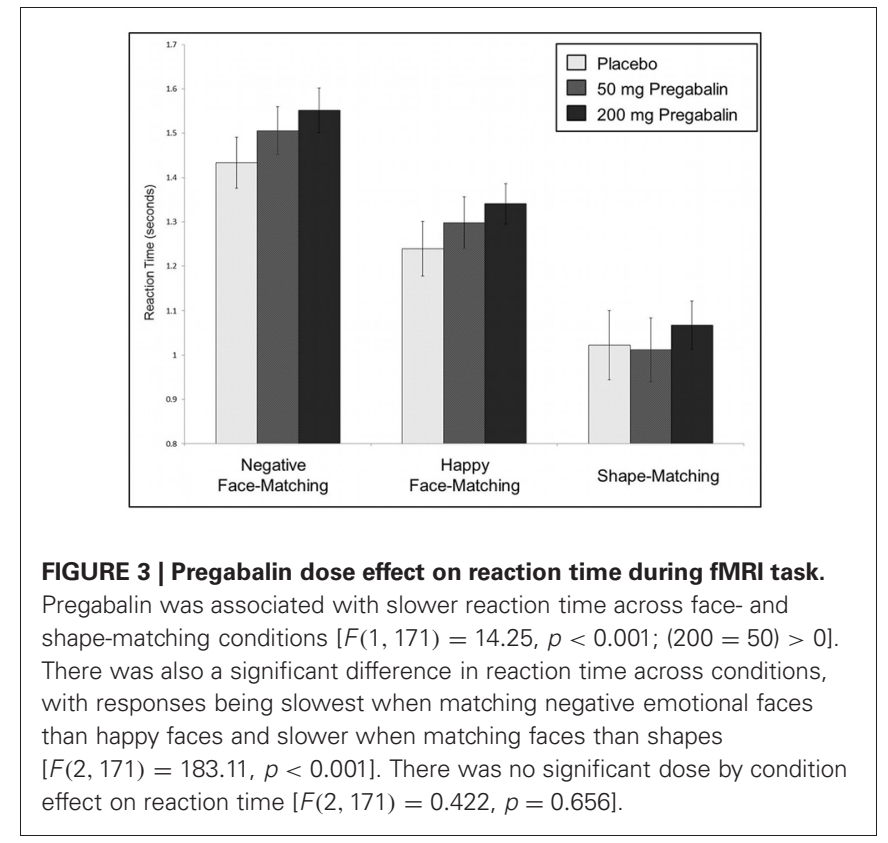

gyrus (BA 37), bilateral amygdala/parahippocampal gyrus, and one region of the subcallosal ACC (BA 25). Happy face-matching was associated with greater activation than fearful face-matching within the ACC/medial PFC (BA 32,10). All of these regions, except the subcallosal ACC cluster (exhibiting Fear > Happy for ROI analyses) and the insula clusters remained significant for whole-brain voxel-wise analysis (see Table $\mathbf{1}$ for full list of whole-brain results and statistics).

Within ROIs, angry face-matching was associated with greater activation than happy face-matching within the bilateral fusiform gyrus (BA 37), bilateral amygdala and PHG, and bilateral anterior/mid insula (BA 13). Happy face-matching was associated with greater activation than angry within the medial PFC/ACC (BA 10,32). All of these clusters remained significant for the whole-brain voxel-wise analysis. See Table 2 for full list of wholebrain results and statistics. See Figure 4 for task effect results within region of interests.

\section{Pregabalin effects on BOLD response to emotional faces}

There were no clusters for region of interest or whole-brain analyses that reached significance for the dose by valence interaction effect when comparing either fear vs. happy or angry vs. happy emotional face-matching. Thus, results focus on dose main effects for each condition type (fearful, angry, and happy face-matching).

PGB effects during fearful face-matching. Region of interest analyses. Region of interest analyses revealed that for fearful face-matching, pregabalin decreased activations within the left fusiform gyrus [BA 37; PLB > $(50=200)]$ and left amygdala (192 ul; PLB > 200).

Whole-brain analyses. Whole-brain analyses revealed that $200 \mathrm{mg}$ pregabalin also increased activations within the right lateral middle frontal (BA 8) and right culmen/lingual gyrus area (BA 18,19), while the $50 \mathrm{mg}$ dose seemed to have unique effects 
Table 1 | Task effect for fMRI emotional face matching task: Fear vs. Happy.

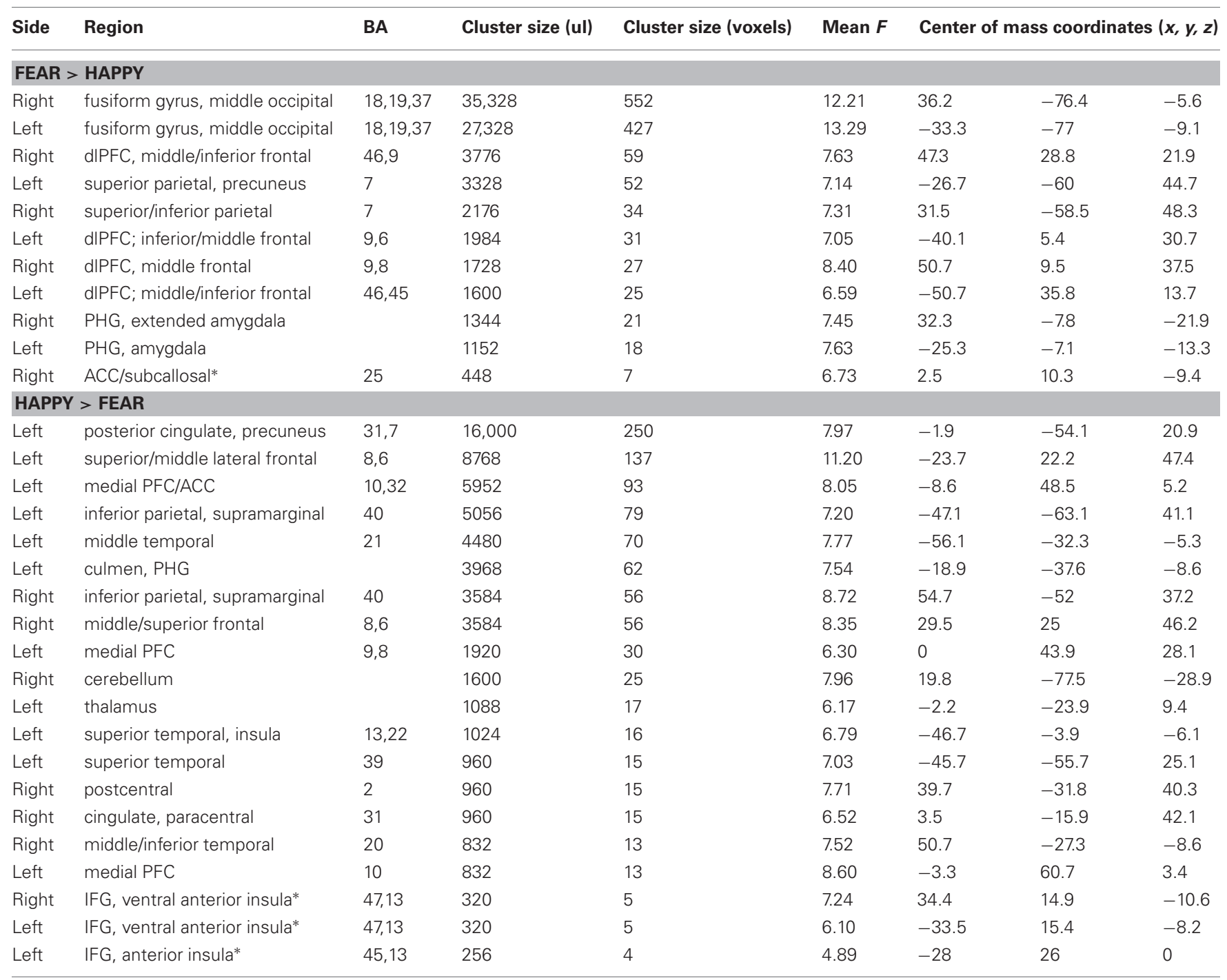

Regions in which there was a main effect of valence (Fear vs. Happy) on BOLD response during the emotional face matching task.

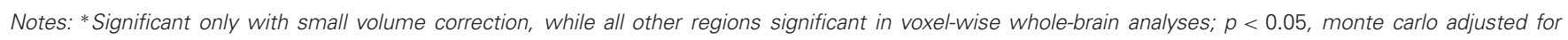
multiple comparisons. All coordinates are Talairach coordinates $(x, y, z)$ based on Talairach Daemon software [Lancaster et al. (2000)]. One voxel is equal to 4 mm ${ }^{3}$.

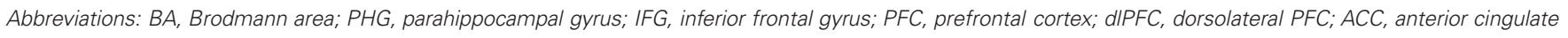
cortex.

from the $200 \mathrm{mg}$ dose and was associated with decreased activations within left superior temporal (BA 13), right inferior parietal (BA 13), and right lateral and medial frontal cortex (BA 6,8), and increased activation within left middle frontal cortex (BA 46), left ACC (BA 25), and right cerebellum. See Table 3 for a full list of whole-brain voxel-wise results of pregabalin effects on neural responses to fearful face-matching.

A separate analysis with session number as a covariate revealed that, with exception of clusters within the ROI fusiform gyrus (BA $37 ; x, y, z=-36.2,-53.9,-11.8)$ and the left middle frontal cortex (BA 46; x, y, $z=-46.6,17.7,20.4$ ), all clusters remained significant. See Figure 5 for pregabalin dose effect results within regions of interest.
PGB effects during angry face-matching. Region of interest analyses. Region of interest analyses revealed that for angry face-matching, pregabalin decreased activations within the left fusiform gyrus [BA 37; PLB $>(50=200)]$ and the $50 \mathrm{mg}$ dose decreased activations within the right anterior insula [BA 13 ; PLB > 50] and increased activation within a region of the ACC/caudate [BA 25; $50>(0=200)]$.

Whole-brain analyses. Whole-brain analyses revealed that both doses of pregabalin also decreased activations in left anterior insula/IFG area (BA13,47), right superior temporal (BA 22), and left middle temporal cortex (BA 22), and that the $200 \mathrm{mg}$ dose decreased activation within the right dorsal medial frontal cortex (BA 6). The $200 \mathrm{mg}$ dose increased activation, while the $50 \mathrm{mg}$ 
Table 2 | Task effect for fMRI emotional face matching task: Angry vs. Happy.

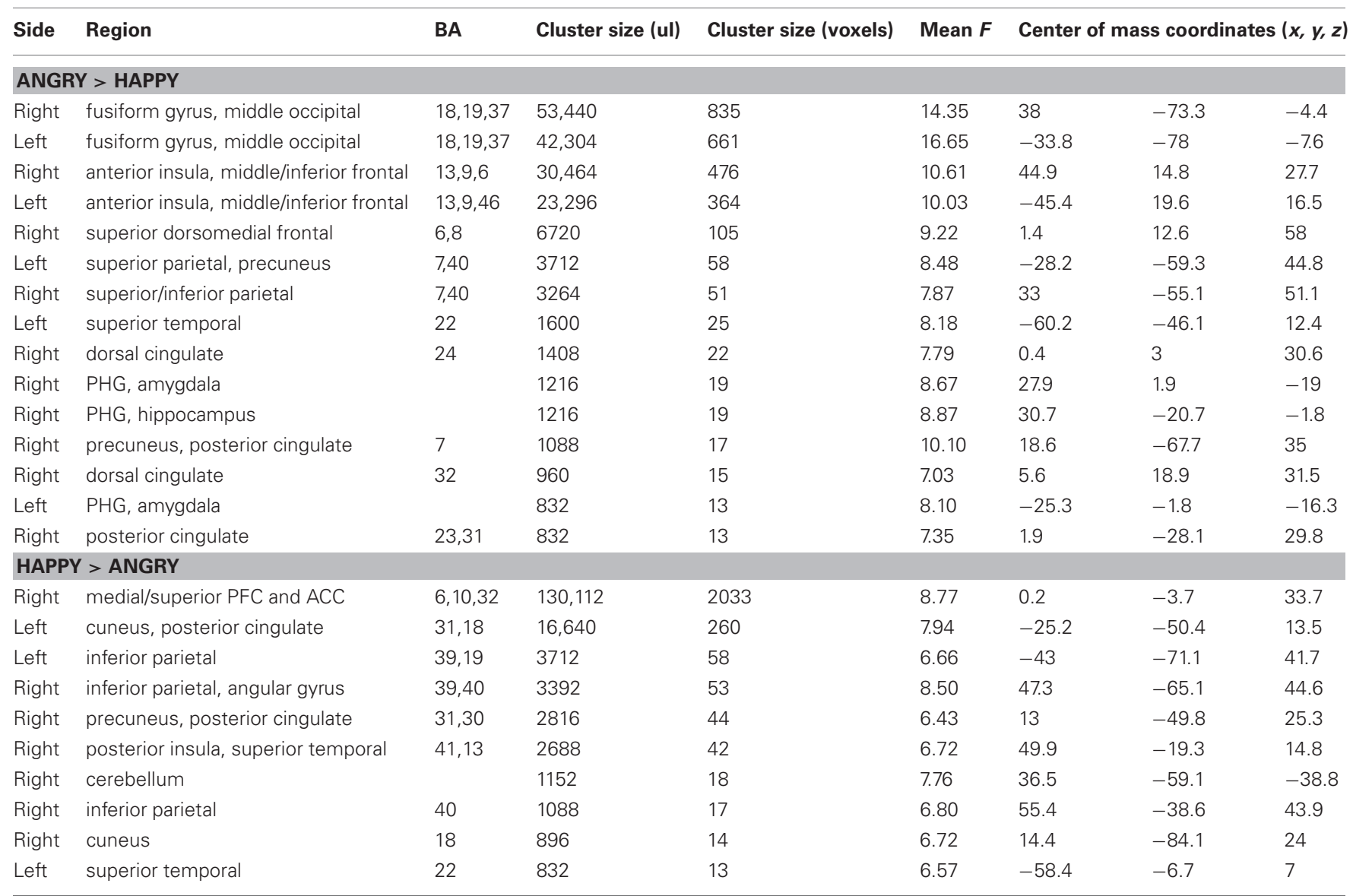

Regions in which there was a main effect of valence (Angry vs. Happy) on BOLD response during the emotional face matching task.

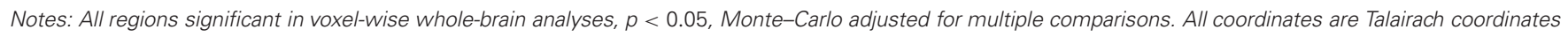

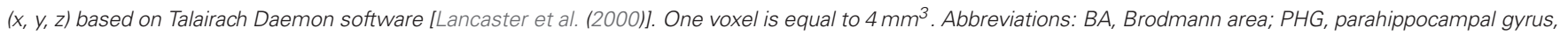
IFG, inferior frontal gyrus, PFC, prefrontal cortex.

dose decreased activation within the left dorsolateral PFC (BA 9). The $50 \mathrm{mg}$ dose was additionally associated with increased activation within left superior parietal (BA 7), right postcentral gyrus (BA 5 and 3), and left dorsal cingulate (BA 24). See Table 4 for a full list of whole-brain voxel-wise results of pregabalin effects on neural responses to angry face-matching.

A separate analysis with session number as a covariate revealed that, with the exception of a cluster within the fusiform gyrus (BA 37; x,y,z=43,55,-16), all clusters remained significant. See Figure 5 for pregabalin dose effect results within regions of interest.

PGB effects during happy face-matching. Region of interest analyses. There were no consistent dose effects identified within ROIs for happy face-matching. The $50 \mathrm{mg}$ dose had the unique effect of increasing activation within the right fusiform gyrus (BA 37) and left medial PFC/ACC (BA 32,10).

Whole-brain analyses. Whole-brain analyses revealed that the $200 \mathrm{mg}$ dose increased activation within the right inferior parietal (BA 40,2) while the $50 \mathrm{mg}$ dose increased activation within the right superior middle frontal (BA 46,10), right middle cingulate
(BA 24,23), left IFG (BA 44), and right cerebellum, and decreased activation within the right middle/inferior temporal (BA 37,19). The $50 \mathrm{mg}$ pregabalin dose increased activation within the left superior middle frontal (BA 10,11) while the $200 \mathrm{mg}$ dose decreased activation in this region. See Table 5 for a full list of whole-brain voxel-wise results.

A separate analysis with session number as a covariate resulted in clusters within the right cerebellum $(x, y, z=$ 8.7, -52, -39.6), left IFG (BA 44; $x, y, z=-41.9,16.7,16.2$ ), right superior middle frontal (BA 46,10; $x, y, z=53.7,44.3,3.4$ ), right middle cingulate (BA 24,23; $x, y, z=4.9,-19,37.6$ ), and middle/inferior temporal cortex (BA 37,19; $x, y, z=$ $47.9,-63.2,2.1)$ to no longer reach significance. All other reported clusters remained significant.

\section{ASL ANALYSIS}

Results from LME analysis investigating effects of pregabalin on $\mathrm{CBF}$ have been reported previously for this cohort (Aupperle et al., 2011) and showed PGB to be associated with increased CBF within the left ventral ACC only $[F(2,24)=6.803, p<$ $0.005,-12,40,2$; post-hoc test revealed $50 \mathrm{mg}>\mathrm{PLB}]$. CBF was 


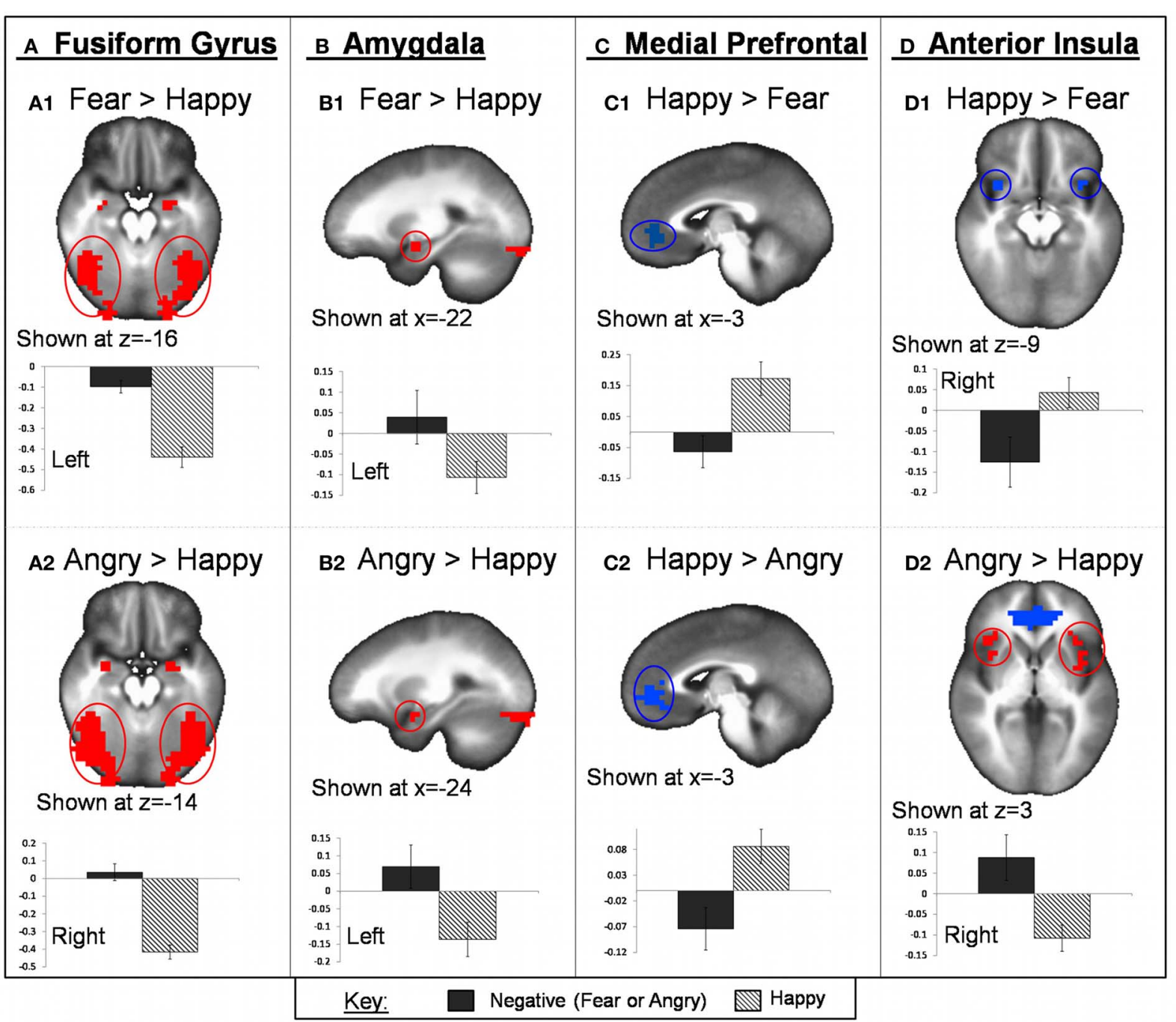

FIGURE 4 | Task valence effect on fMRI BOLD activation. Regions within bilateral fusiform gyrus (A) and bilateral amygdala (B) showed greater activation for both fearful and angry faces compared to happy. Bilateral anterior insula also showed greater activation for angry compared to happy faces (D2) but showed greater activation to happy compared to fearful faces (D1). Regions of the MPFC exhibited greater activation for happy compared to both angry and fearful faces (C). Graphs depicted below each image represent average PSC for negative (fearful or angry) and happy conditions. extracted for each cluster identified as significant in the fMRI ROI analyses discussed above to investigate correlations with fMRI PSC. For those ROI clusters identified as being significantly influenced by dose effect for angry, fear, or happy face-matching, there were no correlations between ASL, CBF, and PSC (all $p$-values > $0.100)$.

\section{fMRI BOLD AND BEHAVIORAL MEASURES}

To determine whether pregabalin effects on BOLD activation in ROIs related to its effects on subjective ratings or task behavior, correlational analyses were conducted with extracted PSC for the ROI activation clusters discussed previously and each behavioral and self-report measure also shown to be influenced by pregabalin. For happy face-matching, there were negative correlations between change in VAS ratings of "trembling" (post-scan - pre-dosing) and the mid ACC (BA 32,10; $384 \mathrm{ul}$ ) cluster from ROI analyses ( $r h o=-0.328, p=0.023$ ). For fearful face-matching, there was a negative correlation between change in VAS ratings of "trembling" and the amygdala cluster (rho = $-0.394, p=0.006)$. These results indicate that subjects who were experiencing greater feelings of trembling, exhibited less neural activation in these regions. For angry face-matching, there were no correlations between ROI clusters of activation and behavioral or self-report measures (all $p$-values $<0.100$ ).

\section{DISCUSSION}

Pregabalin was found to dose-dependently decrease left amygdala response to fearful faces and left anterior insula/inferior frontal gyrus response to angry faces. These effects are similar to previous studies reporting benzodiazepines and SSRIs to decrease 
Table 3 | Pregabalin dose effect on fMRI emotional face matching task: Fear Faces.

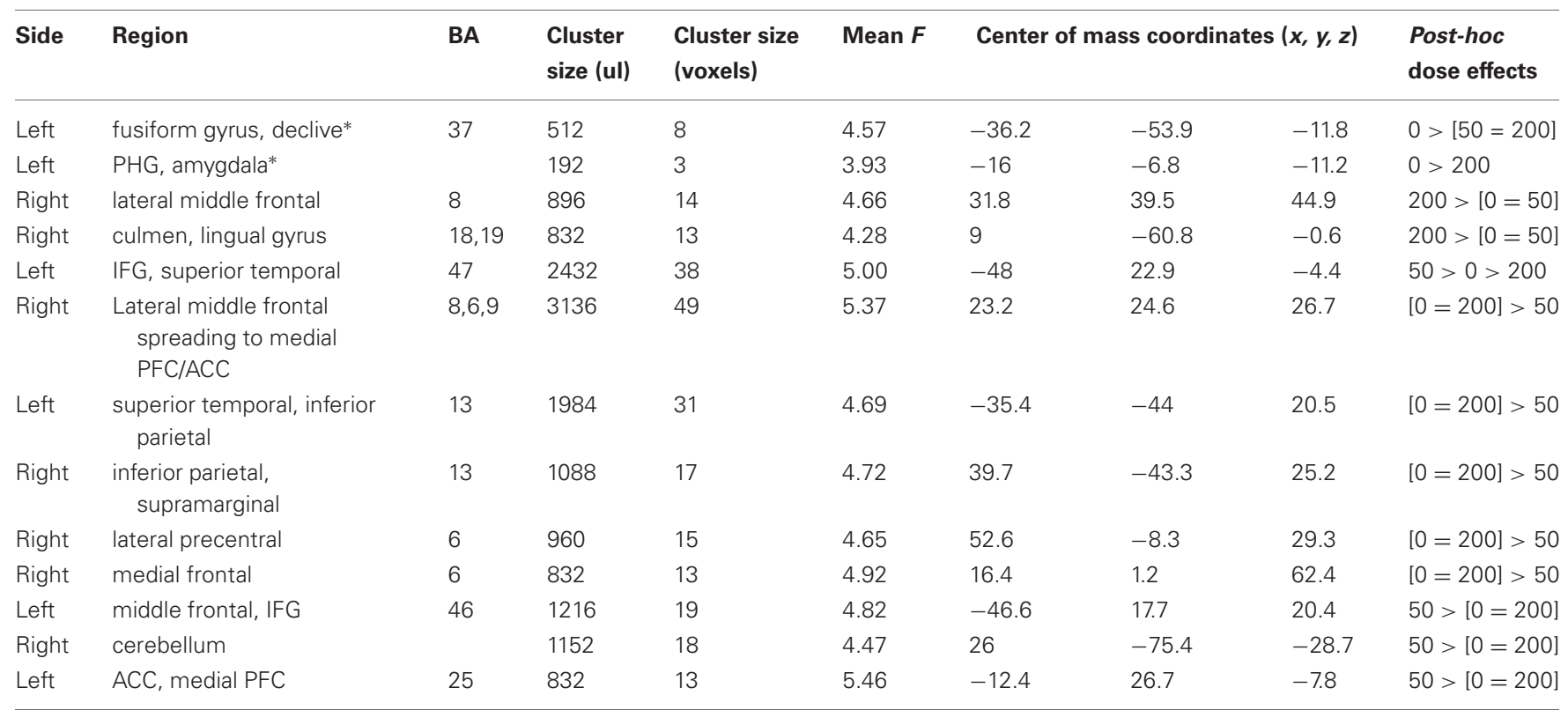

Regions in which there was a pregabalin dose main effect on BOLD response during trials in which subjects were matching fearful emotional faces.

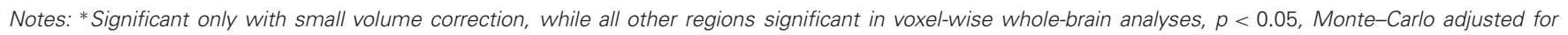

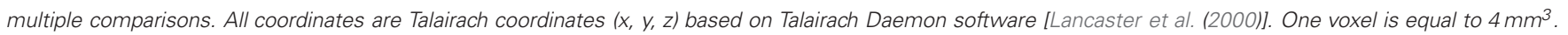
Abbreviations: BA, Brodmann area; PHG, parahippocampal gyrus; IFG, inferior frontal gyrus; PFC, prefrontal cortex; ACC, anterior cingulate cortex.

Table 4 | Pregabalin dose effect on fMRI emotional face matching task: Angry Faces.

\begin{tabular}{|c|c|c|c|c|c|c|c|c|c|}
\hline \multirow{2}{*}{$\begin{array}{l}\text { Side } \\
\text { Right }\end{array}$} & \multirow{2}{*}{$\begin{array}{l}\text { Region } \\
\text { superior temporal }\end{array}$} & \multirow{2}{*}{$\begin{array}{l}\text { BA } \\
22 \\
\end{array}$} & \multirow{2}{*}{$\begin{array}{l}\begin{array}{l}\text { Cluster } \\
\text { size (ul) }\end{array} \\
3072 \\
\end{array}$} & \multirow{2}{*}{$\begin{array}{l}\begin{array}{l}\text { Cluster size } \\
\text { (voxels) }\end{array} \\
48\end{array}$} & \multirow{2}{*}{$\begin{array}{l}\text { Mean } \boldsymbol{F} \\
5.49\end{array}$} & \multicolumn{3}{|c|}{ Center of mass coordinates $(x, y, z)$} & \multirow{2}{*}{$\begin{array}{l}\begin{array}{l}\text { Post-hoc } \\
\text { dose effects }\end{array} \\
0>[50=200]\end{array}$} \\
\hline & & & & & & 59.5 & -41.5 & 15.4 & \\
\hline Left & IFG, anterior insula & 47,13 & 1216 & 19 & 4.46 & -43.5 & 21.3 & 2.2 & $0>[50=200]$ \\
\hline Right & dorsal medial frontal & 6 & 2304 & 36 & 4.93 & 3.9 & -10.9 & 62.6 & {$[0=50]>200$} \\
\hline Left & postcentral, superior parietal & 7 & 2048 & 32 & 4.76 & -19.9 & -48.7 & 63.9 & $50>[0=200]$ \\
\hline Right & cerebellum & & 1600 & 25 & 4.56 & 4.2 & -48.9 & -40.2 & $50>[0=200]$ \\
\hline Right & postcentral gyrus & 5 & 960 & 15 & 5.24 & 21.2 & -41.9 & 66.6 & $50>[200=0]$ \\
\hline Right & $\begin{array}{l}\text { postcentral/paracentral } \\
\text { gyrus }\end{array}$ & 3 & 832 & 13 & 4.93 & 13.2 & -35.4 & 70.5 & $50>[0=200]$ \\
\hline
\end{tabular}

Regions in which there was a pregabalin dose main effect on BOLD response during trials in which subjects were matching angry emotional faces.

Notes: *Significant only with small volume correction, while all other regions significant in voxel-wise whole-brain analyses, $p<0.05$, Monte-Carlo adjusted for multiple comparisons. All coordinates are Talairach coordinates $(x, y, z)$ based on Talairach Daemon software [Lancaster et al. (2000)]. One voxel is equal to 4 mm ${ }^{3}$. Abbreviations: BA, Brodmann area; PHG, parahippocampal gyrus; IFG, inferior frontal gyrus; PFC, prefrontal cortex; ACC, anterior cingulate cortex.

amygdala and/or insula activation during emotional face processing (McKie et al., 2005; Paulus et al., 2005; Harmer et al., 2006; Anderson et al., 2007; Arce et al., 2008; Windischberger et al., 2010). In addition, they are consistent with our previous report that pregabalin dose-dependently decreases amygdala and insula activation during anticipation of emotional images
(Aupperle et al., 2011). Despite their unique neuronal mechanisms of action, evidence collected from the current and previous studies suggests that benzodiazepines, SSRIs, and pregabalin may share a common regional brain mechanism-namely, the attenuation of insula and/or amygdala activation during emotional processing. This supports the potential for fMRI to be used as a 
Table 5 | Pregabalin dose effect on fMRI emotional face matching task: Happy Faces.

\begin{tabular}{|c|c|c|c|c|c|c|c|c|c|}
\hline \multirow{2}{*}{$\begin{array}{l}\text { Side } \\
\text { Right }\end{array}$} & \multirow{2}{*}{$\begin{array}{l}\text { Region } \\
\text { inferior parietal, postcentral }\end{array}$} & \multirow{2}{*}{$\begin{array}{l}\text { BA } \\
40,2\end{array}$} & \multirow{2}{*}{$\begin{array}{l}\begin{array}{l}\text { Cluster } \\
\text { size (ul) }\end{array} \\
1152\end{array}$} & \multirow{2}{*}{$\begin{array}{l}\text { Cluster size } \\
\text { (voxels) }\end{array}$} & \multirow{2}{*}{$\begin{array}{l}\text { Mean } \boldsymbol{F} \\
4.62\end{array}$} & \multicolumn{3}{|c|}{ Center of mass coordinates $(x, y, z)$} & \multirow{2}{*}{$\begin{array}{l}\begin{array}{l}\text { Post-hoc } \\
\text { dose effects }\end{array} \\
200>[0=50]\end{array}$} \\
\hline & & & & & & 55.6 & -30.2 & 43.8 & \\
\hline Right & cerebellum & & 960 & 15 & 4.14 & 8.7 & -52 & -39.6 & $50>[0=200]$ \\
\hline Left & IFG & 44 & 896 & 14 & 4.78 & -41.9 & 16.7 & 16.2 & $50>[0=200]$ \\
\hline Right & middle cingulate & 24,23 & 832 & 13 & 4.07 & 4.9 & -19 & 37.6 & $50>[0=200]$ \\
\hline Right & fusiform gyrus* & 37 & 448 & 7 & 4.40 & 41.1 & -52 & -14 & $50>[0=200]$ \\
\hline Left & mid ACC, medial PFC* & 32,10 & 384 & 6 & 3.95 & -4 & 46.7 & 1.3 & $50>[0=200]$ \\
\hline Right & middle/inferior temporal & 37,19 & 960 & 15 & 4.78 & 47.9 & -63.2 & 2.1 & {$[200=0]>50$} \\
\hline
\end{tabular}

Regions in which there was a pregabalin dose main effect on BOLD response during trials in which subjects were matching happy emotional faces.

Notes: * Significant only with small volume correction, while all other regions significant in voxel-wise whole-brain analyses, $p<0.05$, Monte-Carlo adjusted for multiple comparisons. All coordinates are Talairach coordinates $(x, y, z)$ based on Talairach Daemon software [Lancaster et al. (2000)]. One voxel is equal to 4 mm ${ }^{3}$. Abbreviations: BA, Brodmann area; PHG, parahippocampal gyrus; IFG, inferior frontal gyrus; PFC, prefrontal cortex; ACC, anterior cingulate cortex.

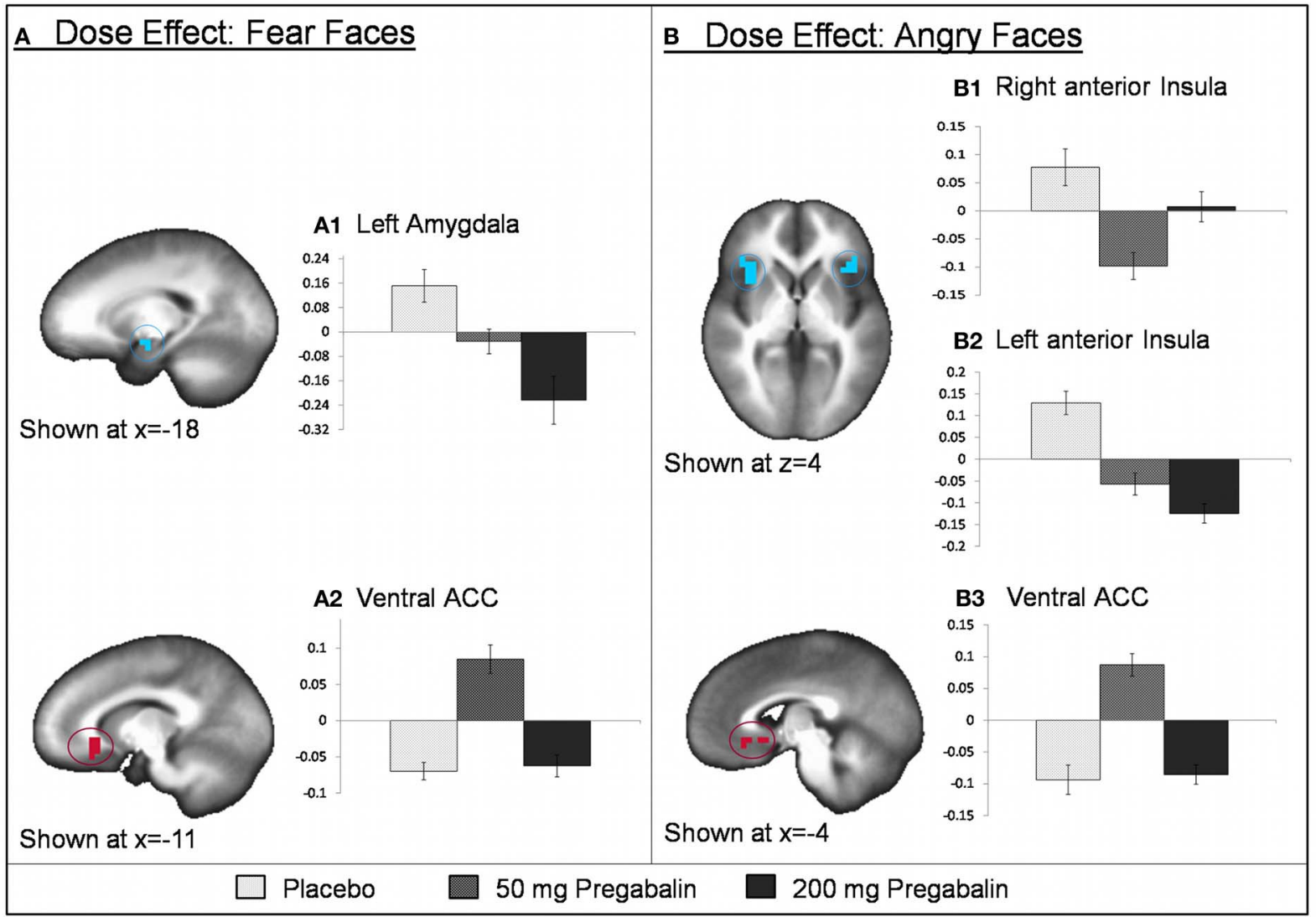

FIGURE 5 | Pregabalin effect on fMRI BOLD activation during processing of fear and angry emotional faces. For fear faces, pregabalin was associated with a consistent decrease in left amygdala $[0>(50=200)$; identified via region of interest (ROI) analyses] activation while $50 \mathrm{mg}$ pregabalin increased ventral ACC activation (identified via whole-brain analyses). For angry faces, pregabalin was associated with a consistent decrease in a region of the left anterior insula/inferior frontal gyrus [B2; $0>(50=200)$; identified via whole-brain analyses] while $50 \mathrm{mg}$ pregabalin decreased right anterior insula activation [B1; $(0=200)>50$; identified via ROI analyses] and increased ventral ACC activation [B3; $50>(0=200)$; identified via ROI analyses]. Graphs depicted with each image represent average PSC for negative emotional faces for each dose (placebo, $50 \mathrm{mg}$, and $200 \mathrm{mg}$ pregabalin). 
biomarker for detecting or predicting anxiolytic effects of current and novel agents and suggest that continued research to further this endeavor is warranted.

It is interesting to note that pregabalin exhibited significant effects on the amygdala during fearful face-matching but on the insula during angry face-matching. This must be considered in the context of our task effect findings, in which angry faces activated anterior insula greater than happy faces whereas the opposite pattern was found for fearful faces. A recent metaanalyses of fMRI findings related to emotional face processing found that while amgydala activation was significant for happy, fearful, and sad faces, insula activation was instead significant for angry and disgust faces (Fusar-Poli et al., 2009). While the amygdala is thought to be important for processing stimuli that are emotionally salient or that have reinforcement value (Davis and Whalen, 2001; Morrison and Salzman, 2010), the insula is considered important for monitoring the internal bodily state and potentially predicting future changes in somatosensory state (Critchley et al., 2004; Craig, 2009). Perhaps seeing angry faces produces increased internal monitoring or hypervigilance, i.e., to examine if there is anything "wrong," whereas fearful faces instead provoke more of an external hypervigilance. Given that the insula and amygdala may play different roles during processing of emotional stimuli and situations, it is potentially important that pregabalin (and other anxiolytics) influences responses within both of these regions in response to relevant contexts. On the other hand, if future pharmacologic agents were able to selectively target specific emotional processes and brain regions, treatment may be able to be tailored for different types of anxiety symptoms.

Perhaps the most surprising finding in the current study was that the $50 \mathrm{mg}$ dose of pregabalin seemed to exhibit more robust effects than the $200 \mathrm{mg}$ dose in many different regions. For example, the $50 \mathrm{mg}$ dose increased responses within the ventral ACC for both angry and fearful faces and decreased activation within the right anterior insula to angry faces, while the $200 \mathrm{mg}$ dose seemed to normalize responses in these regions to placebo levels. Notably, the clinical literature suggests that while $200 \mathrm{mg}$ doses of PGB are effective in the treatment of anxiety (Feltner et al., 2003; Pohl et al., 2005), doses of 50-150 mg have minimal clinical benefit (Feltner et al., 2003; Pande et al., 2004). The $50 \mathrm{mg}$ dose was therefore not expected to exhibit stronger effects on neural activation than the $200 \mathrm{mg}$ dose. One could postulate that the $50 \mathrm{mg}$ dose, while not clinically beneficial for anxiety patients, is a more appropriate "anxiolytic" dose for the healthy control subjects used in this study. However, these explanations are unlikely to be the primary reason for these findings given that more consistent pregabalin dose effects were found for this same cohort and same dosing levels using an emotional anticipation paradigm (Aupperle et al., 2011). The inconsistent dose effects could also relate to there being a "just right" phenomenon concerning pregabalin dosing, with $50 \mathrm{mg}$ somehow producing neural effects that are "too much" and do not produce as much clinical benefit as the more tempered effects of the $200 \mathrm{mg}$ dose. These results could also point to a potential disconnect between dose-dependent effects of pregabalin on clinical symptoms and effects on brain activation. Notably, the emotional face processing paradigm used in the current study is thought to be most sensitive to amygdala function (Hariri et al., 2002; Adolphs, 2008; Fusar-Poli et al., 2009; Staugaard, 2010) and pregabalin exhibited a consistent dose effect on amygdala response to fearful faces. Thus, it may be that attenuation of amygdala response is the most relevant biomarker for this specific paradigm, with effects on other regions being less clinically relevant. Future research examining the relationship between acute or sub-chronic brain effects (during various emotional processing paradigms) and longer-term clinical effects of pregabalin in the same population is needed to clarify the dose effects found in the current study.

The current study had several limitations. First, it investigated neural effects of acute PGB administration. Clinical trials with pregabalin have reported symptom reduction within as early as $4 \mathrm{~h}$ post-administration (Feltner et al., 2003). However, it is possible that acute and chronic doses of pregabalin have different effects on neural response patterns. Future investigations regarding neural effects of chronic pregabalin would be useful in extending the current findings. The strength of the current study is that it examined the effects of different doses of PGB as compared to placebo in the same group of subjects. However, this could also be considered a limitation in that it required repeated administration of the same anticipation paradigm and, therefore, could have been influenced by habituation or sensitization effects. Although the ROI results reported remained largely unchanged when including session as a covariate, there remains the potential that repeated testing influenced results. In addition, the subjects included in this study were healthy controls without any psychiatric diagnosis. Individuals with anxiety disorders have been shown to exhibit greater activation within insula and amygdala regions compared to healthy controls during emotional face processing tasks (Stein et al., 2002, 2007; Shin et al., 2005). We would, therefore, expect that the effects of pregabalin on activation within these regions may be even more pronounced than in the current study. However, given that many of the dose effects observed in this study were inconsistent with what is known regarding clinical dosing with pregabalin, it is possible that pregabalin's effects differ depending on the psychological state of the individual. Future research investigating pregabalin effects on neural activation of anxiety disorder patients is needed to clarify the clinical implications of the current findings and the viability of using fMRI to characterize and predict anxiolytic treatment effects.

\section{CONCLUSIONS}

The current study found pregabalin to dose-dependently decrease left insula and amygdala responses during processing of negative emotional faces, similar to what was found previously for anticipation of emotional images. This lends support to the notion that fMRI may prove to be a useful biomarker for characterizing and predicting anxiolytic potential of pharmacologic agents. However, pregabalin also produced doseinconsistent effects within insula, anterior cingulate, and other regions, in which the $50 \mathrm{mg}$ dose seemed to have a more robust effect than the more clinically-relevant $200 \mathrm{mg}$ dose. This indicates that further research is needed to determine the clinical relevance of pharmaco-fMRI findings to different dosings of 
anxiolytic agents and to healthy control versus anxiety disorder populations.

\section{ACKNOWLEDGMENTS}

We would like to thank Kun $\mathrm{Lu}$ at the UCSD Imaging Center for his help with reconstruction of ASL images, Susan Tapert

\section{REFERENCES}

Adolphs, R. (2008). Fear, faces, and the human amygdala. Curr. Opin. Neurobiol. 18, 166-172.

Akerstedt, T., and Gillberg, M. (1990). Subjective and objective sleepiness in the active individual. Int. J. Neurosci. 52, 29-37.

Anderson, I. M., Del-Ben, C. M., McKie, S., Richardson, P., Williams, S. R., Elliott, R., and Deakin, J. F. (2007). Citalopram modulation of neuronal responses to aversive face emotions: a functional MRI study. Neuroreport 18, 1351-1355.

Arce, E., Miller, D. A., Feinstein, J. S., Stein, M. B., and Paulus, M. P. (2006). Lorazepam dosedependently decreases risk-taking related activation in limbic areas. Psychopharmacology (Berl.) 189, 105-116.

Arce, E., Simmons, A. N., Lovero, K. L., Stein, M. B., and Paulus, M. P. (2008). Escitalopram effects on insula and amygdala BOLD activation during emotional processing. Psychopharmacology (Berl.) 196, 661-672.

Atack, J. R. (2003). Anxioselective compounds acting at the GABA(A) receptor benzodiazepine binding site. Curr. Drug Targets CNS Neurol. Disord. 2, 213-232.

Aupperle, R. L., and Paulus, M. P. (2010). Neural systems underlying approach and avoidance in anxiety disorders. Dialogues Clin. Neurosci. 12, 305-319.

Aupperle, R. L., Ravindran, L., Tankersley, D., Flagan, T., Stein, N. R., Simmons, A. N., Stein, M. B., and Paulus, M. P. (2011). Pregabalin influences insula and amygdala activation during anticipation of emotional images. Neuropsychopharmacology 36, 1466-1477.

Baldwin, D. S. (2008). Room for improvement in the pharmacological treatment of anxiety disorders. Curr. Pharm. Des. 14, 3482-3491.

Bereza, B. G., Machado, M., and Einarson, T. R. (2009). Systematic review and quality assessment of economic evaluations and qualityof-life studies related to generalized anxiety disorder. Clin. Ther. 31, 1279-1308.

Botvinick, M. M. (2007). Conflict monitoring and decision making: reconciling two perspectives on anterior cingulate function. Cogn Affect. Behav. Neurosci. 7, 356-366.

Brawek, B., Loffler, M., Dooley, D. J., Weyerbrock, A., and Feuerstein, T. J. (2008). Differential modulation of $\mathrm{K}(+)$-evoked (3) $\mathrm{H}$-neurotransmitter release from human neocortex by gabapentin and pregabalin. Naunyn Schmiedebergs Arch. Pharmacol. 376, 301-307.

Cannistraro, P. A., and Rauch, S. L. (2003). Neural circuitry of anxiety: evidence from structural and functional neuroimaging studies. Psychopharmacol. Bull. 37, 8-25.

Cloos, J. M., and Ferreira, V. (2009). Current use of benzodiazepines in anxiety disorders. Curr. Opin. Psychiatry 22, 90-95.

Cox, R. W. (1996). AFNI: software for analysis and visualization of functional magnetic resonance neuroimages. Comput. Biomed. Res. 29 162-173.

Craig, A. D. (2009). How do you feel-now? The anterior insula and human awareness. Nat. Rev. Neurosci. 10, 59-70.

Craig, A. D. (2011). Significance of the insula for the evolution of human awareness of feelings from the body. Ann. N.Y. Acad. Sci. 1225, 72-82.

Critchley, H. D., Wiens, S., Rotshtein, P., Ohman, A., and Dolan, R. J. (2004). Neural systems supporting interoceptive awareness. Nat. Neurosci. 7, 189-195.

Davis, M., and Whalen, P. J. (2001). The amygdala: vigilance and emotion. Mol. Psychiatry 6, 13-34. Delvai, N. A., Williams, S. R., Elliott, R., Dolan, M., and Anderson, I. M. (2005). The effect of citalopram pretreatment on neuronal responses to neuropsychological tasks in normal volunteers: an fMRI study. Neuropsychopharmacology 30, 1724-1734.

Dooley, D. J., Taylor, C. P., Donevan, S., and Feltner, D. (2007). Ca2+ channel alpha2delta ligands: novel modulators of neurotransmission. Trends Pharmacol. Sci. 28, 75-82.

Ebner, K., and Singewald, N. (2006). The role of substance $\mathrm{P}$ in stress and anxiety responses. Amino Acids 31, 251-272.
Del-Ben, C. M., Deakin, J. F., McKie, S.,

for advice on post-processing analysis of ASL data, and Alan Simmons for helpful comments on the manuscript.

\section{FUNDING}

Funding for this project provided by National Institute of Mental Health grants MH65413 and MH64122.

Etkin, A., Egner, T., and Kalisch, R. (2011). Emotional processing in anterior cingulate and medial prefrontal cortex. Trends Cogn. Sci. (Regul. Ed.) 15, 85-93.

Etkin, A., and Wager, T. D. (2007) Functional neuroimaging of anxiety: a meta-analysis of emotional processing in PTSD, social anxiety disorder, and specific phobia. Am. J. Psychiatry 164, 1476-1488.

Feltner, D. E., Crockatt, J. G., Dubovsky, S. J., Cohn, C. K., Shrivastava, R. K., Targum, S. D., Liu-Dumaw, M., Carter, C. M., and Pande, A. C. (2003). A randomized, double-blind, placebo-controlled, fixed-dose, multicenter study of pregabalin in patients with generalized anxiety disorder. J. Clin. Psychopharmacol. 23, 240-249.

Fink, K., Dooley, D. J., Meder, W. P. Suman-Chauhan, N., Duffy, S. Clusmann, H., and Gothert, $\mathrm{M}$ (2002). Inhibition of neuronal $\mathrm{Ca}(2+)$ influx by gabapentin and pregabalin in the human neocortex. Neuropharmacology 42, 229-236.

Floyd, T. F., Ratcliffe, S. J., Wang, J. Resch, B., and Detre, J. A. (2003) Precision of the CASL-perfusion MRI technique for the measurement of cerebral blood flow in whole brain and vascular territories. J. Magn. Reson. Imaging 18, 649-655.

Fusar-Poli, P., Placentino, A., Carletti, F., Landi, P., Allen, P., Surguladze, S., Benedetti, F., Abbamonte, M., Gasparotti, R., Barale, F., Perez, J., Mcguire, P., and Politi, P. (2009). Functional atlas of emotional faces processing: a voxel-based meta-analysis of 105 functional magnetic resonance imaging studies. J. Psychiatry Neurosci. 34 418-432.

Gordon, J. A., and Hen, R. (2004) The serotonergic system and anxiety. Neuromolecular Med. 5 27-40.

Hariri, A. R., Tessitore, A., Mattay, V. S., Fera, F., and Weinberger, D. R. (2002). The amygdala response to emotional stimuli: a comparison of faces and scenes. Neuroimage 17, 317-323.

Hariri, A. R., and Whalen, P. J. (2011). The amygdala: inside and out F1000 Biol. Rep. 3, 2.
Harmer, C. J., Mackay, C. E., Reid, C. B., Cowen, P. J., and Goodwin, G. M. (2006). Antidepressant drug treatment modifies the neural processing of nonconscious threat cues. Biol. Psychiatry 59, 816-820.

Hindmarch, I., Trick, L., and Ridout, F. (2005). A double-blind, placeboand positive-internal-controlled (alprazolam) investigation of the cognitive and psychomotor profile of pregabalin in healthy volunteers. Psychopharmacology (Berl.) 183 133-143.

Hoffman, D. L., Dukes, E. M., and Wittchen, H. U. (2008). Human and economic burden of generalized anxiety disorder. Depress. Anxiety 25, 72-90.

Hofmann, S. G., Ellard, K. K., and Siegle, G. J. (2011). Neurobiological correlates of cognitions in fear and anxiety: a cognitive-neurobiological information-processing model. Cogn. Emot. 26, 282-299.

Johnston, K., Westerfield, W., Momin, S., Phillippi, R., and Naidoo, A. (2009). The direct and indirect costs of employee depression, anxiety, and emotional disorders-an employer case study. J. Occup. Environ. Med. 51, 564-577.

Kalk, N. J., Nutt, D. J., and LingfordHughes, A. R. (2011). The role of central noradrenergic dysregulation in anxiety disorders: evidence from clinical studies. J. Psychopharmacol. 25, 3-16.

Kanske, P., Heissler, J., Schonfelder, S., Bongers, A., and Wessa, M. (2010). How to regulate emotion? Neural networks for reappraisal and distraction. Cereb. Cortex. 21, 1379-1388.

Kessler, R. C., Chiu, W. T., Demler, O., Merikangas, K. R., and Walters, E. E. (2005). Prevalence, severity, and comorbidity of 12-month DSM-IV disorders in the National Comorbidity Survey Replication. Arch. Gen. Psychiatry 62, 617-627.

Kim, M. J., Loucks, R. A., Palmer, A. L., Brown, A. C., Solomon, K. M., Marchante, A. N., and Whalen, P. J. (2011). The structural and functional connectivity of the amygdala: from normal emotion to pathological anxiety. Behav. Brain Res. 223, 403-410. 
Knutson, B., and Greer, S. M. (2008). Anticipatory affect: neural correlates and consequences for choice. Philos. Trans. R. Soc. Lond. B Biol. Sci. 363, 3771-3786.

Lancaster, J. L., Woldorff, M. G., Parsons, L. M., Liotti, M., Freitas, C. S., Rainey, L., Kochunov, P. V., Nickerson, D., Mikiten, S. A., and Fox, P. T. (2000). Automated Talairach atlas labels for functional brain mapping. Hum. Brain Mapp. 10, 120-131.

Lowry, C. A., Johnson, P. L., HaySchmidt, A., Mikkelsen, J., and Shekhar, A. (2005). Modulation of anxiety circuits by serotonergic systems. Stress 8, 233-246.

Lydiard, R. B. (2003). The role of GABA in anxiety disorders. J. Clin. Psychiatry 64(Suppl. 3), 21-27.

Lydiard, R. B., Rickels, K., Herman, B., and Feltner, D. E. (2010). Comparative efficacy of pregabalin and benzodiazepines in treating the psychic and somatic symptoms of generalized anxiety disorder. Int. J. Neuropsychopharmacol. 13, 229-241.

Matsumoto, A., and Ekman, A.M., (1988). Japanese and Caucasian Facial Expressions of Emotion (IACFEE) [Slides]. San Francisco: Intercultural and Emotion Research Laboratory, Department of Psychology, San Francisco State University.

McIntosh, A., Cohen, A., Turnbull, N., Esmonde, L., Dennis, P., Eatock, J., Feetam, C., Hague, J., Hughes, I., Kelly, J., Kosky, N., Lear, G., Owens, L., Ratcliffe, J., and Salkovskis, P. (2004). Clinical Guidelines and Evidence Review for Panic Disorder and Generalised Anxiety Disorder. Sheffield: University of Sheffield/London: National Collaborating Center for Primary Care.

McKie, S., Del-Ben, C., Elliott, R., Williams, S., Del Vai, N., Anderson, I., and Deakin, J. F. (2005). Neuronal effects of acute citalopram detected by pharmacoMRI. Psychopharmacology (Berl.) 180, 680-686.

Millan, M. J. (2003). The neurobiology and control of anxious states. Prog. Neurobiol. 70, 83-244.

Montgomery, S. A., Tobias, K., Zornberg, G. L., Kasper, S., and Pande, A. C. (2006). Efficacy and safety of pregabalin in the treatment of generalized anxiety disorder: a 6-week, multicenter, randomized, double-blind, placebo-controlled comparison of pregabalin and venlafaxine. J. Clin. Psychiatry 67, 771-782.

Morrison, S. E., and Salzman, C. D. (2010). Re-valuing the amygdala. Curr. Opin. Neurobiol. 20, 221-230.

Nutt, D., Mandel, F., and Baldinetti, F. (2008). Early onset anxiolytic efficacy after a single dose of pregabalin: double-blind, placeboand active-comparator controlled evaluation using a dental anxiety model. J. Psychopharmacol. 23, 867-873.

Ochsner, K. N., and Gross, J. J. (2005). The cognitive control of emotion. Trends Cogn. Sci. 9, 242-249.

Oulis, P., Konstantakopoulos, G. Kouzoupis, A. V., Masdrakis, V. G., Karakatsanis, N. A., Karapoulios, E., Kontoangelos, K. A., and Papadimitriou, G. N. (2008). Pregabalin in the discontinuation of long-term benzodiazepines' use. Hum. Psychopharmacol. 23, 337-340.

Pande, A. C., Crockatt, J. G., Feltner, D. E., Janney, C. A., Smith, W. T., Weisler, R., Londborg, P. D., Bielski, R. J., Zimbroff, D. L., Davidson, J. R., and Liu-Dumaw, M. (2003). Pregabalin in generalized anxiety disorder: a placebo-controlled trial. Am. J. Psychiatry 160, 533-540.

Pande, A. C., Feltner, D. E., Jefferson, J. W., Davidson, J. R., Pollack, M. Stein, M. B., Lydiard, R. B., Futterer, R., Robinson, P., Slomkowski, M., Duboff, E., Phelps, M., Janney, C. A., and Werth, J. L. (2004). Efficacy of the novel anxiolytic pregabalin in social anxiety disorder: a placebo-controlled, multicenter study. J. Clin. Psychopharmacol. 24, 141-149.

Paulus, M. P., Feinstein, J. S., Castillo, G., Simmons, A. N., and Stein, M. B. (2005). Dose-dependent decrease of activation in bilateral amygdala and insula by lorazepam during emotion processing. Arch. Gen. Psychiatry 62, 282-288.

Paulus, M. P., and Stein, M. B. (2006). An insular view of anxiety. Biol. Psychiatry 60, 383-387.

Paulus, M. P., and Stein, M. B. (2007). Role of functional magnetic resonance imaging in drug discovery. Neuropsychol. Rev. 17, 179-188.

Pirkola, S., Saarni, S., Suvisaari, J., Elovainio, M., Partonen, T., Aalto, A. M., Honkonen, T., Perala, J., and Lonnqvist, J. (2009). General health and quality-of-life measures in active, recent, and comorbid mental disorders: a populationbased health 2000 study. Compr. Psychiatry 50, 108-114.

Pohl, R. B., Feltner, D. E., Fieve, R. R., and Pande, A. C. (2005). Efficacy of pregabalin in the treatment of generalized anxiety disorder: double-blind, placebo-controlled comparison of BID versus TID dosing. J. Clin. Psychopharmacol. 25 151-158.

Rack-Gomer, A. L., Liau, J., and Liu, T. T. (2009). Caffeine reduces restingstate BOLD functional connectivity in the motor cortex. Neuroimage 46, 56-63.

Ravindran, L. N., and Stein, M. B. (2010). The pharmacologic treatment of anxiety disorders: a review of progress. J. Clin. Psychiatry 71, 839-854.

Rickels, K., Pollack, M. H., Feltner, D. E., Lydiard, R. B., Zimbroff, D. L., Bielski, R. J., Tobias, K., Brock, J. D., Zornberg, G. L., and Pande, A. C. (2005). Pregabalin for treatment of generalized anxiety disorder: a 4-week, multicenter, double-blind, placebo-controlled trial of pregabalin and alprazolam. Arch. Gen Psychiatry 62, 1022-1030.

Shin, L. M., and Liberzon, I. (2010) The neurocircuitry of fear, stress, and anxiety disorders. Neuropsychopharmacology 35, 169-191.

Shin, L. M., Wright, C. I., Cannistraro, P. A., Wedig, M. M., Mcmullin, K., Martis, B., Macklin, M. L., Lasko, N. B., Cavanagh, S. R., Krangel, T. S., Orr, S. P., Pitman, R. K., Whalen, P. J., and Rauch, S. L. (2005). A functional magnetic resonance imaging study of amygdala and medial prefrontal cortex responses to overtly presented fearful faces in posttraumatic stress disorder. Arch. Gen. Psychiatry 62, 273-281.

Simmons, A. N., Arce, E., Lovero, K. L., Stein, M. B., and Paulus, M. P. (2009). Subchronic SSRI administration reduces insula response during affective anticipation in healthy volunteers. Int. J. Neuropsychopharmacol. 12, 1009-1020.

Spielberger, C. D., Gorsuch, R. L., Lushene, P. R., Vagg, P. R., and Jacobs, A. G. (1983). Manual for the State-Trait Anxiety Inventory (Form Y). Palo Alto: Consulting Psychologists Press, Inc.

Staugaard, S. R. (2010). Threatening faces and social anxiety: a literature review. Clin. Psychol. Rev. 30, 669-690.

Stein, M. B., Goldin, P. R., Sareen, J., Zorrilla, L. T., and Brown, G. G. (2002). Increased amygdala activation to angry and contemptuous faces in generalized socia phobia. Arch. Gen. Psychiatry 59, 1027-1034.

Stein, M. B., Simmons, A. N., Feinstein, J. S., and Paulus, M. P. (2007)
Increased amygdala and insula activation during emotion processing in anxiety-prone subjects. Am. J. Psychiatry 164, 318-327.

Takahashi, H., Yahata, N., Koeda, M., Takano, A., Asai, K., Suhara, T., and Okubo, Y. (2005). Effects of dopaminergic and serotonergic manipulation on emotional processing: a pharmacological fMRI study. Neuroimage 27, 991-1001.

Tassone, D. M., Boyce, E., Guyer, J., and Nuzum, D. (2007). Pregabalin: a novel gamma-aminobutyric acid analogue in the treatment of neuropathic pain, partial-onset seizures, and anxiety disorders. Clin. Ther. 29, 26-48.

Tottenham, N., Tanaka, J. W., Leon, A. C., Mccarry, T., Nurse, M., Hare, T. A., Marcus, D. J., Westerlund, A., Casey, B. J., and Nelson, C. (2009). The NimStim set of facial expressions: judgments from untrained research participants. Psychiatry Res. 168, 242-249.

Vaswani, M., Linda, F. K., and Ramesh, S. (2003). Role of selective serotonin reuptake inhibitors in psychiatric disorders: a comprehensive review. Prog. Neuropsychopharmacol. Biol. Psychiatry 27, 85-102.

Windischberger, C., Lanzenberger, R., Holik, A., Spindelegger, C., Stein, P., Moser, U., Gerstl, F., Fink, M., Moser, E., and Kasper, S. (2010). Area-specific modulation of neural activation comparing escitalopram and citalopram revealed by pharmaco-fMRI: a randomized cross-over study. Neuroimage 49 , 1161-1170.

Wise, R. G., Lujan, B. J., Schweinhardt, P., Peskett, G. D., Rogers, R., and Tracey, I. (2007). The anxiolytic effects of midazolam during anticipation to pain revealed using fMRI. Magn. Reson. Imaging 25, 801-810.

Wong, E. C., Buxton, R. B., and Frank, L. R. (1998). A theoretical and experimental comparison of continuous and pulsed arterial spin labeling techniques for quantitative perfusion imaging. Magn. Reson. Med. 40, 348-355.

Conflict of Interest Statement: Robin Aupperle, Lakshmi Ravindran, Dharol Tankersley, Taru Flagan, Murray Stein, and Martin Paulus do not report any direct conflicts of interest related to this manuscript. Dr. Stein has received compensation as a consultant for various companies involved in pharmaceuticals for psychiatric disorders (see full listing of disclosures below), including Pfizer, which distributes Lyrica (pregabalin). 
Dr. Aupperle receives or has in the past three years: Salary/funding from the VA San Diego Healthcare System, University of California, San Diego, and University of Missouri, Kansas City. Dr. Ravindran receives or has in the past three years: Salary/funding from the VA San Diego Healthcare System, University of California, San Diego, and University of Toronto. Dr. Tankersley receives or has in the past three years: Salary/funding from Duke University, University of California, San Diego, and Baylor University.
Ms. Flagan receives or has in the past three years received: Salary/funding from the University of California, San Diego and University of Texas. Dr. Stein receives or has in the past three years: Research funding from Department of Veterans Affairs; US Department of Defense; Hoffmann-La Roche; National Institute of Mental Health. He has been a paid consultant for: AstraZeneca; Bristol-Myers Squibb; Comprehensive NeuroScience; Jazz Pharmaceuticals; Johnson \& Johnson; Pfizer; Sepracor. $\mathrm{He}$ also receives compensation for his work as an editor with Up-to-Date, and Wiley (Depression and Anxiety). Dr. Paulus receives or has in the past three years: Salary/funding from the San Diego VA Healthcare System and the University of California, San Diego; GlaxoSmithKline; Hoffmann-La Roche.

Received: 06 October 2011; accepted: 21 February 2012; published online: 27 March 2012.

Citation: Aupperle RL, Tankersley D, Ravindran LN, Flagan T, Stein NR, Stein
MB and Paulus MP (2012) Pregabalin effects on neural response to emotional faces. Front. Hum. Neurosci. 6:42. doi: 10.3389/fnhum.2012.00042

Copyright (c) 2012 Aupperle, Tankersley, Ravindran, Flagan, Stein, Stein and Paulus. This is an open-access article distributed under the terms of the Creative Commons Attribution Non Commercial License, which permits non-commercial use, distribution, and reproduction in other forums, provided the original authors and source are credited. 